\title{
Applications and Future Perspectives of Virtual Reality in the Treatments of Post Traumatic Stress Disorder
}

\author{
Ancen Lyu \\ Department of Cognitive Science, CA 92093, University of California, San Diego, USA \\ anlyu@ucsd.edu
}

\begin{abstract}
The experience of severe trauma serves as the causation of several mental health disorders. Post Traumatic Stress Disorder (PTSD) is a common example. The traditional treatments for this kind of mental health disorder have achieved some positive effects. Still, they cannot create an isolated and interactive environment, teaching patients how to overcome the disorders by repeatedly simulating the problematic trauma environment. This ability is the advantage of virtual reality (VR) treatments. This paper will focus on the principle of VR treatments for this mental disorder, some examples of practical application through several historical research and experiments, the shortcomings and problems faced by this treatment, and some further thoughts and perspectives about this kind of treatment. The result of this research revealed the significantly positive effects of VR treatment for PTSD and even other related mental disorders. It also shows that VR has an excellent future prospect.
\end{abstract}

\section{CCS CONCEPTS}

- Human-centered computing $\rightarrow$ Human-computer interaction (HCI); Empirical studies in HCI.

\section{KEYWORDS}

Virtual reality (VR), Mental health disorders, PTSD, Treatment

\section{ACM Reference Format:}

Ancen Lyu. 2021. Applications and Future Perspectives of Virtual Reality in the Treatments of Post Traumatic Stress Disorder. In 2021 3rd International Conference on Intelligent Medicine and Image Processing (IMIP '21), April 23-26, 2021, Tianjin, China. ACM, New York, NY, USA, 5 pages. https://doi. org $/ 10.1145 / 3468945.3468970$

\section{INTRODUCTION}

As a kind of treatment for mental disorders that are gradually applied by clinicians, immersive virtual reality (VR) devices have the unique ability to create interactive computer-generated worlds, producing situations that can be therapeutically helpful [1]. VR's function of recreating an immersive environment to simulate the actual situation and exhibiting the complicated situation in patients' brains is especially beneficial for treating mental disorders that could be caused by severe trauma and brain structure deterioration, like PTSD. Several studies have shown the positive results of VR

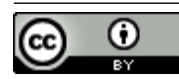

This work is licensed under a Creative Commons Attribution International 4.0 License.

IMIP '21, April 23-26, 2021, Tianjin, China

(C) 2021 Copyright held by the owner/author(s)

ACM ISBN 978-1-4503-9005-7/21/04

https://doi.org/10.1145/3468945.3468970 treatment for PTSD. Figuring out the principles of this kind of PTSD treatment through several examples allows researchers to apply this technology to treat other mental disorders. This innovative method also has the potential to lead to the overall progress of the mental disorders treatment and the medical industry, igniting the innovation of traditional treatment method. Some practical problems and ethical issues that lie behind this VR treatment may also lead to further exploration, development, and application of the VR field.

\section{OVERVIEW OF VR AND POST TRAUMATIC STRESS DISORDER}

VR, as commonly described, is a computer-generated environment or reality that is designed to simulate a person's physical presence in an immersive and convincing environment [2]. Various perceptual devices have been invented to enable users to experience a realistic sensation and perception. Several examples include holography, 3D display, and Head-mounted display(HMD), which has a close relationship with immersive VR.

Among all the essential elements and advantages of VR, immersion grabs the attention of many researchers. Immersion usually focuses on the user's involvement and interaction with the created virtual environment, which means that immersion is better when users receive less perceptual sensation from the real world.

This immersive feature of VR offers it tremendous potential to be applied in the clinical treatments of several mental disorders, especially the disorders that may be caused by a past traumatic experience like PTSD, since VR owns the ability to simulate an environment that patients have difficulties to face, allowing patients to learn to react in this environment appropriately. Clinicians can repeat this procedure without a time limit until the patients overcome their problems facing such an environment or stimulus. So far, VR is the only treatment that could achieve this immersion. VR also has the unique ability to simulate the complex environment of human's brain and nervous system, enabling clinicians to thoroughly learn the details of the patients' brains to figure out the problematic regions. Thus, clinicians could easily figure out the most efficient treatment corresponds to the unique situation of each patient. Additionally, unlike reality, VR can be operated. This feature effectively avoids the real harm that reality may cause to the patients. Patients will also feel psychologically comforted during VR treatment since they know that the situation is not real even though they are in an immersive and realistic environment.

PTSD is often described as a kind of severe and ongoing emotional reaction to psychologically traumatic events like someone's death or the war scene, characterized by intrusive symptoms. Common examples of these symptoms include nightmares, flashbacks, depression, insomnia, numbing feelings, and emotional detachment. 
Although combat-related traumas are studied the most an often regarded as the major causation of PTSD, several types of traumatic experience include robbery, sexual abuse, or even car accidents are all likely to trigger PTSD. Different from traumatic stress, PTSD causes lingering and more intensive traumatic stress. A large number of people are suffering or have suffered from PTSD. In the United States, about $3.5 \%$ of adults have PTSD in a given year, and $9 \%$ of people develop it at some point in their life [3], and these patients sometimes also exhibit other combined psychiatric disorders like addictions, phobia, or bipolar disorder. Therefore, figuring out the effective ways to alleviate the suffering caused by PTSD will contribute a lot to psychotherapy.

\section{CURRENT SITUATION}

Researchers have shown that PTSD involves pathological fear structures that are activated when information represented in the structures is encountered [4]. The most significant advantage of VR used for treating PTSD is its capability to simulate a virtual, interactive, and naturalistic environment, simulating the stimuli that evoke the fear and modifying pathological fear structures. This is partly because patients' memories undergo reconsolidation when VR technology helps them retrieve their fearful memories. Memory is not permanent. During the process of reconsolidation, patients' brains choose to form a more or less permanent memory for certain events. Their memories are vulnerable and malleable during this process. This kind of adaptive malleability provides them with an opportunity to modify or correct some inappropriate response towards certain stimulus. Thus, clinicians could help them eliminate their fear response. PTSD symptoms have shown resistance towards traditional drug and psychotherapy. However, in the noninvasive and safe environment created by VR technology, clinicians can confront the patients, teaching patients to appropriately respond to the traumatic events and alleviating their phobic symptoms.

Since the traditional treatment sometimes fails to reduce the patients' severe symptoms after the therapy, a number of researchers have investigated the use of Virtual Reality Exposure Therapy (VRET) in the treatment of PTSD, although there are still some uncertainties. The earliest studies focused on veterans with chronic PTSD. 21 Vietnam veterans' PTSD symptoms were below baseline by the 3-month post-treatment assessment [5]. Many of such researches yielded a significant reduction in PTSD of veterans. Further researchers have shown that VRET is clinically meaningful for the treatment of different types of trauma. The systematic review of VRET studies also revealed the potential efficacy of this PTSD treatment. Among the six studies that included a control group, a statistically significant reduction in questionnaires evaluating symptoms of PTSD was observed in four [6]. Recent studies showed that VRET is an effective treatment for most combat-related PTSD. All treatment gains were maintained at three- and six-month follow-up [7]. There are also researchers that identified three different virtual trauma intervention approaches, namely, VRET, 3MDR (multi-modular motion-assisted memory desensitization and reconsolidation), and ACET (action-centered exposure therapy) [8]. The result revealed that not only VRET but also other kinds of virtual interventions might also play an influential role in treating PTSD. These kinds of studies may lead the new direction of using

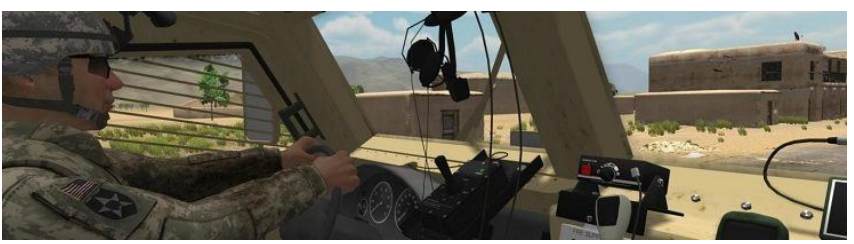

Figure 1: VR for treatment of veterans' PTSD [9]

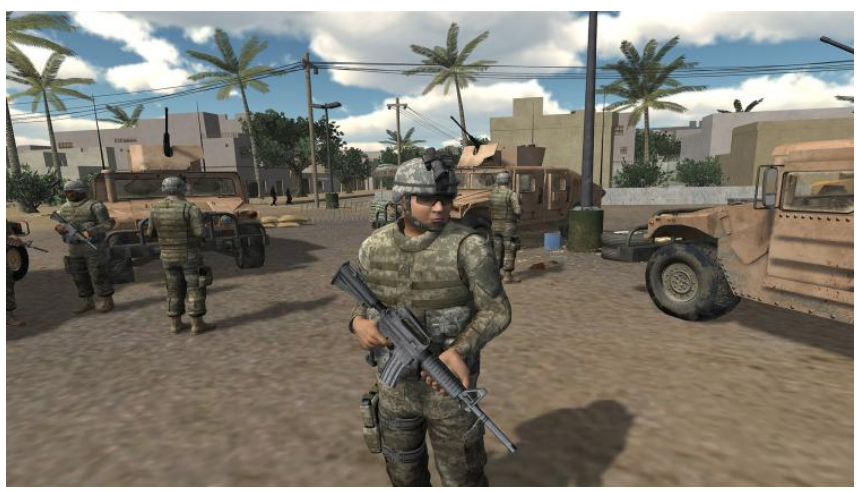

Figure 2: Enter a virtual war zone to relieve the trauma [10]

VR technology to treat PTSD. Figure 1 and figure 2 describe the virtual environment of war scenes created by VR during the PTSD treatment.

\section{FUTURE PERSPECTIVES}

The potential of VR to be used in the treatment of PTSD is not limited to improving patients' situations through simulating the traumatic experience. Although PTSD is mainly caused by the traumatic experience, its reflections include the deterioration and dysfunction of patients' brains and nervous systems. The normal biochemical changes in patients' brains caused by PTSD include an abnormally high level of catecholamine and high concentrations of corticotropin-releasing factor. The abnormally high or low levels of dopamine and the overreactions of the norepinephrine system are also found in PTSD patients. PTSD sometimes can also be distinguished by the over response of insular cortex as the decreased activity of medial prefrontal cortex and hippocampus. Genetics is also a common risk factor for PTSD, and this reveals that figuring out the changes of the nervous system's structures and functions plays an essential role in the process of diagnosing and treating PTSD. One effective way to achieve this goal is to use VR technology.

The traditional scanning technology used to show the inner situation of patients' brains includes CT and MRI (magnetic resonance imaging). Technologies like EEG (electroencephalography) and PET (Positron emission tomography) are also used. However, those technologies lack the ability to vividly simulate and reveal the details in patients' brains in a more immersive way. VR has this capability. If clinicians can combine VR technology with those scanning technologies, like figure 3 indicates, the data acquired through those technologies could be represented as a 3D stereo image. Thus, 


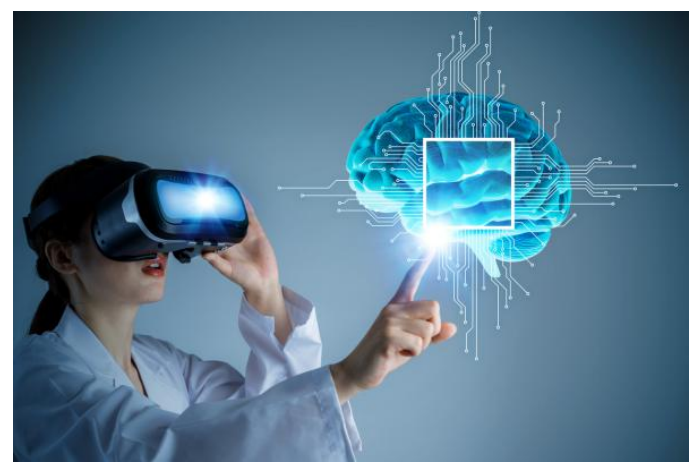

Figure 3: VR with brain-based computer interface [13]

clinicians could observe the structures of the patients' brains and find out the problematic brain regions more thoroughly. For example, PTSD is sometimes accompanied by the reduced volume of hippocampus or a smaller anterior cingulate cortex, and such reductions are commonly associated with traumatic experience like childhood abuse. The advanced VR method allows clinicians to analyze each patient's body situation in a far more detailed way. For instance, when the patient expresses the complaint of pain over the right flank, the physician can rotate the image, make various layers transparent, and query the representation of the patient's liver or kidney [11]. Thus, VR could significantly optimize the diagnosis and treatment of PTSD since the deterioration happened in patients' brains contributes a lot to the symptoms. Clinicians could even simulate the changes of some biochemical reactions' parameters, like the level of specific chemical substances or the number of some receptors, in brains to simulate the effects of possible drugs in a virtual environment, better figuring out the ways to treat their patients.

Since the risk factors and symptoms of various mental disorders share a significant overlap, the VR treatment used for PTSD can also be used to improve the treatments of other mental disorders. For instance, approximately half of people with PTSD also suffer from MDD (major depressive disorder) [12]. This comorbidity has different explanations, but no matter this is due to the imprecise diagnosis or genetic and biological reasons, the fact of this comorbidity suggests that the major symptoms of MDD and PTSD share a lot of similarities. Thus, it is reasonable to use VR to treat MDD like PTSD, modifying patients' emotional and negative responses to the traumatic experience by simulating the traumatic environment. VR experience could provide patients with limitless chances to learn the appropriate response, like training their social skills to reduce their isolation. Actually, although still at an early stage, researchers have already started to apply VR to treat MDD like PTSD. Many experiments of VR treatment for MDD, as well as other mental disorders like schizophrenia, have already made considerable progress.

To be more specific, the risk factors of MDD include several traumatic experiences like physical, sexual, and psychological childhood abuse. Although still at an early stage, several types of research has revealed the efficiency and potential of VR to treat MDD. CBT (cognitive behavioral therapeutic) techniques for the treatment of
MDD can be made into VR experience includes behavioral activation or social skill training. VR offered some unique experiences like pet interactions which improved the patients' situation efficiently. Currently, VR behavioral activation has been used in some MDD cases during the global pandemic. The results revealed that subjects' depression decreased after this VR treatment.

As for schizophrenia, traumatic experiences like childhood trauma also serve as important risk factors. Such traumas could cause nervous system's dysfunctions, resulting in schizophrenia with symptoms like delusions, social withdrawal, hallucination, and disorganized motor behaviors. Several kinds of research have revealed the efficiency of VR to treat schizophrenia, with VR treatment group usually perform better than conventional group in cognitive functioning as well as behaviors like social interaction.

The application of VR in the field of PTSD treatment has the potential to lead the wide application of VR in the treatment of mental disorders and the innovation of mental disorders' treating method.

\section{DISCUSSION}

VR has the unique ability to create a virtual environment to help clinicians treat PTSD like figure 4 shows. However, although having been repeatedly exhibiting positive results, VR treatment for PTSD is still at an early stage and can not completely replace the traditional treatment due to some problems.

As indicated by figure 5, one of the technical problems that need to be solved is the problem of dizziness, which is mainly caused by the mismatch between the movement in our visual perception and the actual movement of our body and brain. VR treatment will become more practicable if researchers can solve the problem of dizziness. Another well-known problem is the sense of isolation. Researchers should still try to alleviate patients' feelings of isolation during the progress of VR treatment. HCI (human-computer interaction) technology is also an obstacle to the application of VR to treat PTSD. Recent VR devices are still far from the convenient and effective interaction method between patients and the virtual world. The combination of different human-machine interaction ways like vocal control and touch control, or even directly getting information from patients' brains using advanced brain-computer interfaces, will ignite the further innovation of VR application in treating PTSD.

The technical problems could be solved in the future through the combined effort from various fields including physiology and computer science. Deeper physiological exploration of human's brain and nervous system will allow researchers to better understand the causation of dizziness as well as other sickness experienced in the virtual environment, leading to a more practicable and comfortable VR device used for patients. Better computer science and human-computer interaction technology will lead to a more advanced system to be used in the treatment of mental disorders like PTSD.

Additionally, researchers are also faced with some ethical and philosophical problems. For instance, in what ways can clinicians make sure that patients could distinguish virtual environments from reality since the ambiguity between reality and imagination itself is already a common symptom of several mental disorders. 


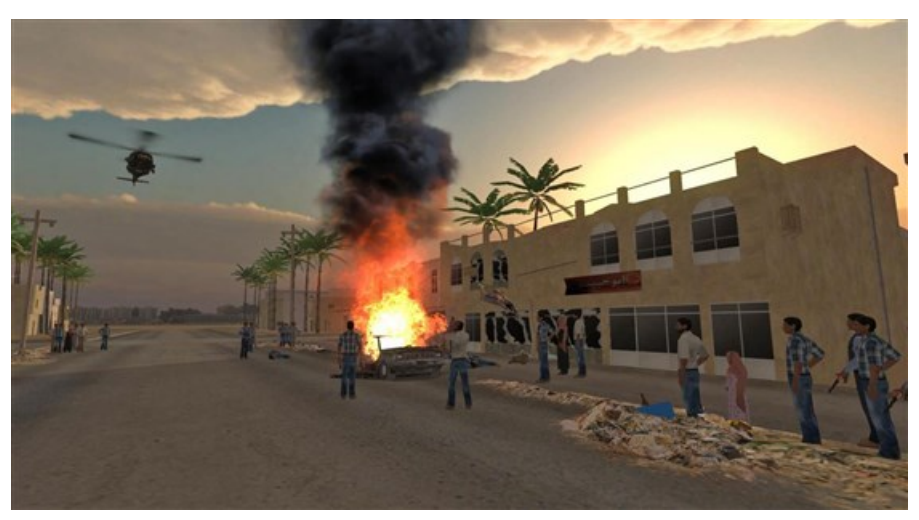

Figure 4: Recreated memory about an explosion in the virtual world [14]

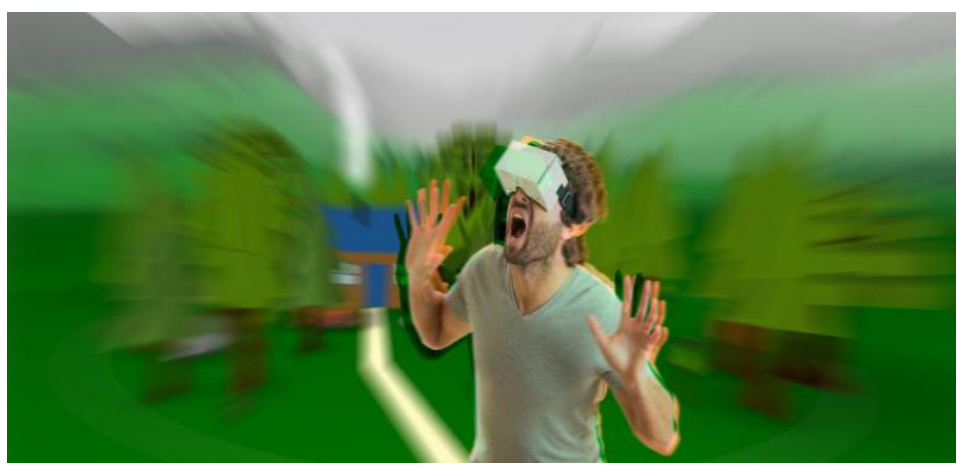

Figure 5: Some people feel sickness in the virtual environment [15]

For instance, delusion, some false and groundless beliefs, and hallucinations, like some internally generated perceptual experiences, are common symptoms of schizophrenia. VR has the potential to increase the ambiguity between reality and virtual world. This may cause patients to suffer more from this situation. Furthermore, current brain exploration shows that mental disorders like PTSD are often accompanied by the changes of the brain structures. Thus, clinicians also need to consider if VR therapy will change the patients' brain structures and results in some unpredictable side effects.

Moreover, what is the appropriate way to improve patients' social skills and to "teach patients to communicate with others" ? Is there an absolute formula for communication between humans? Who should set the standards for this kind of social skills and behaviors training? Those questions are still worth further research and exploration.

\section{CONCLUSION}

Several kinds of Research have revealed the current efficiency and future potential of VR as an effective technology that can be used to treat PTSD due to its strong ability to simulate an immersive environment. This ability enables patients to gradually learn the appropriate responses in a particular traumatic environment or at a specific situation and to correct or modify the inappropriate responses, allowing the clinicians to deeply observe and analyze patients' situation. This technology could optimize the progress of treatment, and this method can also be applied to the treatment of other mental disorders or other kinds of diseases. It also owns the potential to be combined with some traditional technology like scanning technology to improve the diagnosis process and treatment effects. Various types of research about VR treating PTSD as well as other related mental disorders have gotten considerable results. Those also lead to diverse sub-branches of VR treatment technology. Researchers are now trying hard to explore this method and extend this method to the treatment of other mental disorders or even physical disorders. Although still at an early stage and faces many difficulties about technology and ethics, VR treatment for PTSD is showing tremendous potential, and it is expected to have a bright future with the effort from different related fields.

\section{ACKNOWLEDGMENTS}

I want to express my thankfulness to Jane Zhang who gave me suggestions about my research and essay. I also want to express my thankfulness to professor Adam Blumenthal for his instructions and suggestion about virtual reality and its application.

\section{REFERENCES}

[1] Freeman, D., Reeve, S., Robison, A., Ehlers, A., Clark, D., Spanlang, B., Slater, M. (2017).Virtual reality in the assessment, understanding, and treatment of mental health disorders. Psychological Medicine (2017). 47. pp 2393-2400. doi:10.1017/S003329171700040X 
[2] Wolfartsberger, J. (2019). Analyzing the potential of Virtual Reality for engineering design review. www.elsevier.com/locate/autcon

[3] American Psychiatric Association. (2013). Diagnostic and Statistical Manual of Mental Disorders(5th ed). Arlington, VA: American Psychiatric Publishing. pp.271-80

[4] Rizzo, A.S., Shilling, R. (2017). Clinical Virtual Reality tools to advance the prevention, assessment, and treatment of PTSD. European Journal of Psychotraumatology, 8:sup5, 1414560. https://doi.org/10.1080/20008198.2017.1414560.

[5] Ready, D.J., Pollack, S., Rothbaum, B.O., Alarcon, R.D. (2008). Virtual Reality Exposure for Veterans with Posttraumatic Stress Disorder. pp 199-220

[6] Goncalves, R., Pedrozo, A.L., Coutinho, E.S.F., Figueira, I., Ventura P. (2012). Efficacy of Virtual Reality Exposure Therapy in the Treatment of PTSD: A Systematic Review. https://doi.org/10.1371/journal.pone.0048469

[7] Beidel, D.C., Frueh, B.C., Neer, S.M., Bowers, C.A., Trachik,B., Uhde, T.W., Grubaugh, A. (2017). Journal of Anxiety Disorder. Trauma management therapy with virtual-reality augmented exposure therapy for combat-related PTSD: A randomized controlled trial. http://dx.doi.org/10.1016/j.janxdis.2017.08.005

[8] Knaust, T., Felnhofer, A., Kothgassner, O.D., Höllmer, H., Gorzka, R.J., Schulz, H. (2020). Virtual trauma interventions for the treatment of post-trauma stress disorder: a scoping review. doi: 10.3389/fpsyg.2020.562506
[9] Perlman, C. (2017). Bravemind demonstrates how virtual reality can transform mental health treatments through its innovative PTSD exposure therapy system. https://digital.hbs.edu/platform-digit/submission/bravemind-using-virtualreality-to-treat-ptsd/

[10] Parkin, S. (2017). How virtual reality is helping heal soldiers with PTSD. https://www.nbcnews.com/mach/innovation/how-virtual-reality-helpingheal-soldiers-ptsd-n733816

[11] Satava, R.M., Jones, S.B. (1998). Current and future applications of virtual reality for medicine. Proceedings of the IEEE. pp 484-489

[12] Flory, J.D., Yehuda, R. (2015). Comorbidity between post-traumatic stress disorder and major depressive disorder: alternative explanations and treatment considerations. Dialogues Clin Neurosci. pp141-150. doi: 10.31887/DCNS.2015.17.2/jflory

[13] IEEE Xplore digital library. (2018). Facing Virtual Reality: Improving VR with Brain-based Computer interfaces. https://innovate.ieee.org/innovation-spotlight/ brain-computer-interface-virtual-reality-eeg/

[14] Parkin, S. (2017). How virtual reality is helping heal soldiers with PTSD. https://www.nbcnews.com/mach/innovation/how-virtual-reality-helpingHeal-soldiers-ptsd-n733816 accessed on March 24, 2021

[15] Krause, S. (2016). Will I be sick in VR? https://blog.cospaces.io/will-i-be-sick-invr-dc67cbb8824c accessed on March 24, 2021 